\title{
Endoscopic pancreatic necrosectomy: why scuff when you can flush the muck - make it an easy row to hoe $\square$
}

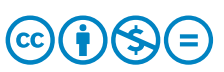

\author{
Authors \\ Rinkesh Kumar Bansal, Rajesh Puri, Narendra S. Choudhary, Sumit Bhatia, Nisharg Patel, Saurabh K. Patle, Gaurav K. \\ Patil, Amit Agarwal, Chandra Prabha, Randhir Sud
}

Institution

Institute of Digestive and Hepatobiliary Sciences, Medanta

The Medicity, Gurugram, India

submitted 17.5.2017

accepted after revision 22.5.2017

Bibliography

DOI https://doi.org/10.1055/s-0043-112854 |

Endoscopy International Open 2017; 05: E847-E853

(c) Georg Thieme Verlag KG Stuttgart · New York

ISSN 2364-3722

Corresponding author

Dr Rajesh Puri, Institute of Digestive and Hepatobiliary

Sciences, Medanta The Medicity, Sector 38, Gurugram,

Delhi NCR, India, PIN 122001

purirajesh70@gmail.com

\section{ABSTRACT}

Background and aims Endoscopic ultrasound (EUS) guided drainage of symptomatic pancreatic walled-off necrosis (WON) followed by fully covered self-expanding metal stent (FCSEMS) placement offers several advantages such as higher technical success rate and the option of necrosectomy. The aim of this study was to evaluate the safety and efficacy of EUS guided drainage of patients with WON by using FCSEMS and intracavitary lavage with a solution con- taining hydrogen peroxide and adopting a step-up approach.

Methods A prospective open label study was carried out at a single tertiary care center between January 2014 and January 2016. Patients with symptomatic WON who underwent EUS guided drainage followed by FCSEMS placement were included. Primary end points were complete drainage with improvement in symptoms or major adverse events. Secondary end points were minor adverse events related to the procedures.

Results A total of 64 patients (mean age 36 years; 52 males) were included. Technical success was achieved in $100 \%$ of patients and clinical success was achieved in $90.6 \%$. Complete drainage was achieved with FCSEMS alone in 18 (28.1\%), FCSEMS with necrosectomy using lavage in 40 $(62.5 \%)$, FCSEMS with percutaneous drainage (PCD) in 5 (7.8\%), and $1(1.6 \%)$ patient required salvage surgery. The major adverse event was life threatening bleeding in 3 $(4.7 \%)$ patients. Minor adverse events were non-life threatening bleeding in $2(3.1 \%)$ patients and stent migration in $3(4.7 \%)$ patients.

Conclusion EUS guided WON drainage with FCSEMS followed by necrosectomy with lavage using a solution containing hydrogen peroxide as a step-up approach is a minimally invasive and effective method with a high technical and clinical success rate. Patients with solid debris $>40 \%$ need aggressive management.

\section{Introduction}

The severity of acute pancreatitis is classified as mild, moderate or severe depending on the presence or absence of organ failure, and local or systemic complications. Local complications of acute pancreatitis include acute peri-pancreatic fluid collection (APFC), acute necrotic collection (ANC), pancreatic pseudocyst (PPC) and walled-off necrosis (WON) [1].

WON, whether sterile or infected, is characterized by a welldefined capsule around necrotic pancreatic material, usually occurring 4 weeks after initial insult. It contains varying amounts of solid debris. Treatment of WON is indicated when it becomes symptomatic, i. e. abdominal pain, infection, gastric outlet obstruction, or biliary compression [2].

Management of WON is drainage, which can be done via endoscopic, surgical or percutaneous methods. However, endoscopic drainage, especially with endoscopic ultrasound (EUS), is the preferred modality [3-5].

Initially, plastic stents were used for EUS guided drainage of WON; however, the solid component within WON frequently obstructed them, leading to insufficient drainage [6]. This led to the advent of fully covered self-expandable metal stents (FCSEMS), which were used to drain WON with encouraging results as these had a wide caliber; however, an increased risk of 
spontaneous stent migration was present because of their longer length and the absence of lumen-apposing properties $[7,8]$.

Short and biflanged dedicated FCSEMSs such as NAGI (Taewoong Medical, Gyeonggi-do, South Korea), and AXIOS (Boston Scientific Corporation, United States) stents were subsequently used to drain WON with good results as the number of interventions required to resolve WON were significantly lower with these stents compared to conventional long FCSEMS or plastic stents [9-11]. Over time, these short FCSEMS became popular.

Traditionally, after a dedicated FCSEMS insertion, endoscopic intracavitary debridement (direct passage of the endoscope into the cavity with necrosectomy) is used, and is associated with a high rate of complications [12]. Whether these complications can be avoided by adopting a better thought out and more acceptable method of endoscopic necrosectomy, is an issue for research [2]. Intracavitary flushing using a water pump has been described in the literature with good results [13].

In the present study, we evaluated the safety and efficacy of EUS guided WON drainage by FCSEMS placement accompanied by intracavitary lavage using a solution containing hydrogen peroxide and adopting a step-up approach of endoscopic necrosectomy.

\section{Methodology}

The current study is a prospective open label study, conducted at a single tertiary center in North India between January 2014 and January 2016 after approval from the institute's ethics committee. A total of 64 patients were finally included in the study.

The inclusion criteria were all patients $>18$ years with symptomatic WON. EUS guided drainage was performed after obtaining informed consent. Exclusion criteria included age $<18$ years, pregnancy and coagulopathy (international normalized ratio $[I N R]>1.5$, platelets $<50000$ ). Anticoagulant and antiplatelet agents were withheld for 5 days before the procedure (in six patients).

Primary end points were completion of drainage with improvement in symptoms or major adverse events. Secondary end points were minor adverse events related to procedures.

\section{Initial procedure details}

All EUS-guided drainage procedures (Step 1) were performed in an inpatient setting. All procedures were performed with the patient in the left lateral position under propofol sedation administered by an anesthetist. All patients received prophylactic antibiotic ( $1 \mathrm{~g}$ cefoperazone and $1 \mathrm{~g}$ sulbactam, iv), 2 hours before beginning the procedure.

A 3.7-mm channel, linear therapeutic echo-endoscope (GFUCT180; Olympus, Tokyo, Japan) was used to access the WON via the stomach or duodenal wall under fluoroscopic guidance. $\mathrm{CO}_{2}$ was used during the procedure in all of the patients. The WON was assessed for size, wall thickness, interposing collaterals, and percentage of solid debris. The WON cavity was punctured with a 19-gauge EUS needle (Expect Slimline by Boston Scientific, Echotip Ultra by Cook), the fluid sample was aspirated and sent for microbial analysis (Gram stain, culture, and sensitivity). Then a 0.035 -inch stiff guidewire (Jagwire, Straight tip, Boston Scientific) was inserted through the needle into the cavity, and coiling into the cavity was confirmed with fluoroscopy. Over the wire, a 6 F cystotome (Endo-flex $\mathrm{GmbH}$, Dusseldorf, Germany) was used to dilate the puncture site and this was further dilated with a wire guided $6 \mathrm{~mm}$ balloon dilator (Hurricane; Boston Scientific). Then, the stent delivery catheter was advanced over the guidewire across the WON wall, and a dedicated FCSEMS (Nagi; Taewoong Medical, Gyeonggi-do, South Korea) was deployed under endosonographic and fluoroscopic visualization. The Nagi stent used for drainage was short in length ( 20 or $30 \mathrm{~mm}$ ) with $16 \mathrm{~mm}$ caliber.

\section{Post-procedure follow-up and antibiotic protocol}

All patients received prophylactic antibiotic ( $1 \mathrm{~g}$ cefoperazone and $1 \mathrm{~g}$ sulbactam, iv), 2 hours before beginning the procedure and this was continued at 12-hour intervals at the same dose for 48 hours. Patients were assessed after 48 hours. If patients were asymptomatic, they were discharged on oral antibiotics (cefuroxime $500 \mathrm{mg}$ tablet, 12 hourly) for 5 days. For patients who had not responded after 48 hours (rising total leukocyte count (TLC), increase in temperature or heart rate, development of organ dysfunction), antibiotic treatment was upgraded according to the fluid culture and antimicrobial sensitivity reports, and a repeat session of necrosectomy was performed.

Endoscopic necrosectomy (Step 2) was performed by lavage with $1 \mathrm{~L}$ of a solution containing $3 \%$ hydrogen peroxide ( $1: 20 \mathrm{di}$ Iution). Irrigation was done with the aid of a $9.9 \mathrm{~mm}$ diameter gastroscope (GIFH180; Olympus, Tokyo, Japan), which was advanced into the cavity through the FCSEMS and irrigation was performed using a water pump and subsequent lavage carried out. All of the liquid/debris was suctioned from the cyst cavity as well as from the stomach. Necrotic material obstructing the stent lumen, leading to hampered drainage, was removed by a

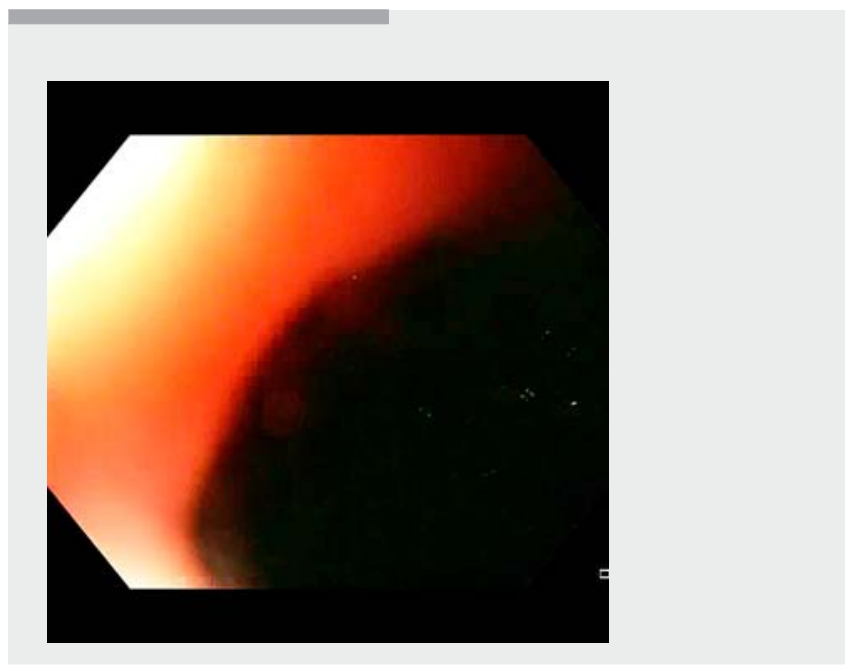

$\checkmark$ Video 1 Endoscopic ultrasound-guided pancreatic necrosectomy using a step-up approach for walled-off pancreatic necrosis. 
snare or Roth net forceps under fluoroscopy. No other debris was removed mechanically ( $\bullet$ Video $\mathbf{1}$ ).

For patients who were symptomatic even after two sessions of lavage (96 hours of FCSEMS placement), a non-contrast CT scan of the abdomen was performed to examine the adequacy of the drainage. In the case of persistence of collection, either repeat necrosectomy or percutaneous drainage (PCD) was performed. In patients in whom there was a separate collection or if it was not amenable to endoscopy, PCD was inserted (Step 3). In patients in whom there was endoscopic amenable collection, a repeat session of necrosectomy was carried out. Repeat endoscopic lavages of the WON were performed every 48 hours (> Fig. 1).

All adverse events such as bleeding, whether life threatening or minor, and stent migration were noted. Massive bleeding can be defined as follows: (i) blood loss exceeding circulating blood volume within a 24 -hour period, (ii) blood loss of $50 \%$ of circulating blood volume within a 3-hour period, (iii) blood loss exceeding $150 \mathrm{~mL} / \mathrm{min}$, or (iv) blood loss that necessitates plasma and platelet transfusion. Torrential bleeding was defined when there was massive bleeding along with rapidly developing hypotension.

\section{Follow-up visits}

Follow-up endoscopy for stent removal was performed 12 weeks after initial drainage after documentation of resolution of WON on non-contrast computed tomography (CT). During removal of FCSEMS, a pancreatogram was obtained under fluoroscopy. If the pancreatogram showed disruption of the pancreatic duct in the head or body region, it was bridged using a plastic stent. If the pancreatic duct appeared disconnected on the pancreatogram, no further action was taken. These patients were prospectively followed over a period of 6 months.

\section{Results}

Our study was a prospective open label study, conducted between January 2014 and January 2016. In total, 78 patients with symptomatic walled-off pancreatic necrosis (WOPN) in the form of abdominal pain, fever, and gastric outlet obstruction leading to failure to thrive, who were amenable to endoscopic drainage, were allocated to EUS guided drainage by FCSEMS. In total, 14 patients were excluded from the study (10 patients did not provide consent, 3 patients had coagulopathy, and 1 patient was pregnant (26 weeks)), so 64 patients (mean age 36 years, range $30-56 ; 52$ male and 12 female) were studied ( $>$ Table 1 ).

The etiology of the pancreatitis leading to WON was alcohol in $46.9 \%(n=30)$, gallstones in $40.6 \%(n=26)$; hypercalcemia in $3.1 \%(n=2)$, and idiopathic in $9.4 \%(n=6)$.

The symptoms that required drainage were fever and abdominal pain in $62.5 \%(n=40)$, recurrent vomiting and failure to thrive in $25 \%(n=16)$, and abdominal pain alone in $12.5 \%(n=$ 8 ). The mean duration of symptoms from the initial event was 36 days (range 30-96 days). Mean initial WON diameter was $12.5 \mathrm{~cm}$ (range $9.0-18.5 \mathrm{~cm}$ ) on CT and/or EUS.
Symptomatic WOPN, amenable to endoscopic drainage $(n=64)$

Step 1 EUS guided FCSEMS placed $(n=64)$

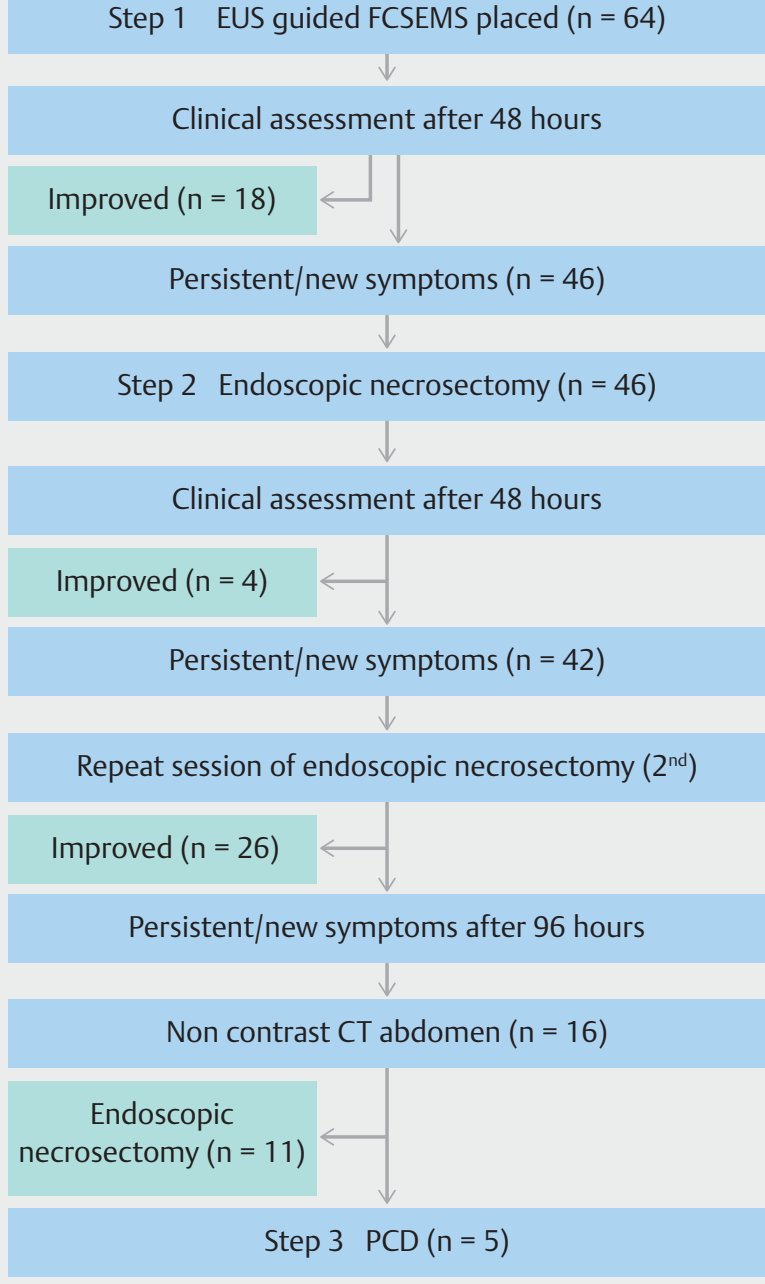

- Fig. 1 Flow diagram showing the study design. FCSEMS, fully covered self-expanding metal stent; PCD, percutaneous drainage; WOPN, walled-off pancreatic necrosis.

Technical success was achieved in $100 \%$ of the patients and clinical success was achieved in $90.6 \%$ of patients $(n=58)$. The primary end point was achieved with FCSEMS alone in $28.1 \%$ $(n=18)$, FCSEMS with necrosectomy in $62.5 \%(n=40)$, and FCSEMS with PCD in $7.8 \%(n=5)$ of patients. In total, $1.6 \%(n=$ 1) of patients required salvage surgery. On subgroup analysis, it was found that patients with more than $40 \%$ solid debris on EUS were likely to need necrosectomy compared to FCSEMS alone (32 (80\%) versus 2 (5\%)).

Mean necrosectomy sessions required were 3.2 (range 1 - 5). Four patients required 1 session, 26 patients 2 sessions, 6 patients 3 sessions, 3 patients 4 sessions, and 1 patient required 5 sessions.

Growth of bacteria in aspirated fluid was detected in $37.5 \%$ $(n=24)$. Sixteen patients showed growth of Escherichia coli and 
- Tab. 1 Demographic profile of the patients and adverse events.

\begin{tabular}{|l|l|}
\hline Age (range), years & $36(30-56)$ \\
\hline Sex, M/F & $52: 12$ \\
\hline $\begin{array}{l}\text { Etiology - Alcoholic/Gallstone/ } \\
\text { Hypercalcemia/Idiopathic, } n\end{array}$ & $30: 26: 2: 6$ \\
\hline $\begin{array}{l}\text { Fever and pain in the abdomen/Recur- } \\
\text { rent vomiting/Pain in the abdomen, } \mathrm{n}\end{array}$ & $40: 16: 8$ \\
\hline Duration of symptoms (range), days & $36(30-96)$ \\
\hline Initial diameter of WON (range), cm & $12.5(9-18.5)$ \\
\hline Technical success, $\mathrm{n}$ (\%) & $64(100)$ \\
\hline Clinical success, $\mathrm{n}$ (\%) & $58(90.6)$ \\
\hline Primary end point, $\mathrm{n}$ & \\
\hline - FCSEMS alone & 18 \\
\hline - FCSEMS with necrosectomy & 40 \\
\hline FCSEMS, fully covered self-expanding metal stent; PCD, percutaneous \\
\hline - FCSEMS with PCD
\end{tabular}

8 showed Klebsiella pneumoniae ssp.pneumoniae. Antibiotics were upgraded according to the antimicrobial sensitivity test.

Average hospital stay was 8.3 days.

\section{Adverse events}

Adverse events were encountered in 8 (12.5\%) patients. Life threatening bleeding was the major event encountered in $4.7 \%$ of patients $(n=3)$. One patient developed torrential bleeding associated with hypotension 2 months after FCSEMS insertion. On CT angiography of the abdomen, there was a pseudoaneurysm of the splenic artery ( $>$ Fig. 2 ) close to the FCSEMS, secondary to impinging and this required salvage surgery, i.e. exploratory laparotomy and ligation of the splenic artery pseudoaneurysm with splenectomy, and the patient survived. He required 8 units of packed red blood cells (PRBC) and 6 units of plasma along with inotropes. Two patients developed massive bleeding (2 months, 2.5 months) after the last session of necrosectomy secondary to pseudoaneurysm of the splenic artery close to the FCSEMS, and which was managed successfully with radiological intervention. One patient (bleeding at 2 months) required 4 units of $P R B C$ while another patient required a total of 5 units of PRBC with 2 units of plasma. There was also impingement of the splenic artery in both of these patients secondary to NAGI stent emplacement.

Minor adverse events were non-life threatening bleeding in $3.1 \%$ of patients $(n=2)$ and stent migration in $4.7 \%(n=3)$. One patient had stent migration to the rectum (> Fig.3) 2 months after the initial procedure, and it was spontaneously

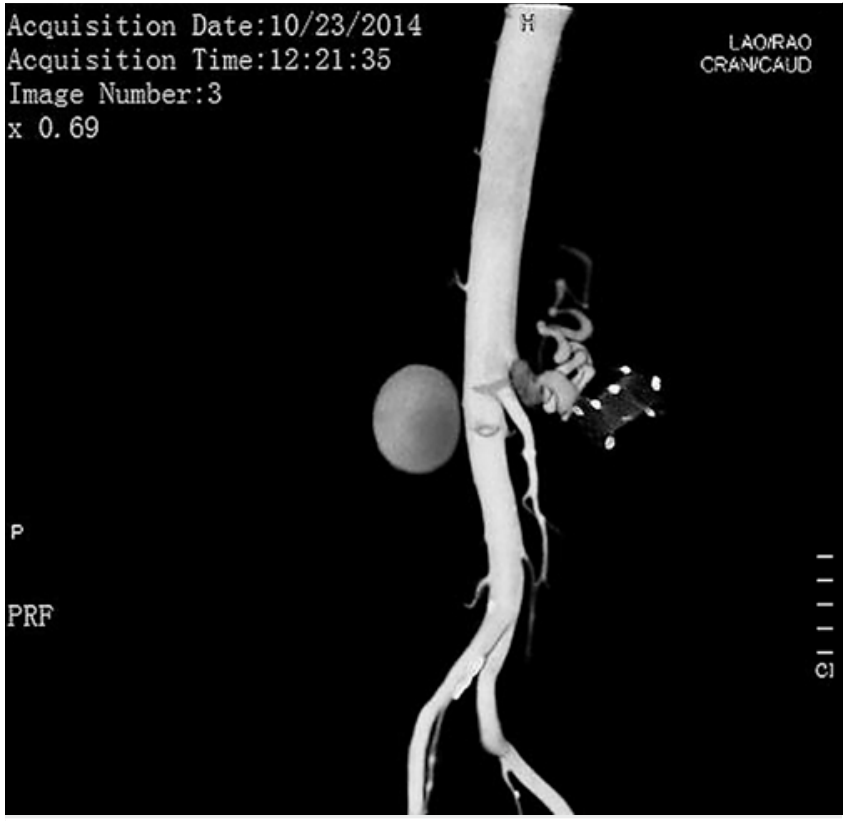

Fig. 2 Splenic artery pseudoaneurysm with FCSEMS on CT angiography of the abdomen.

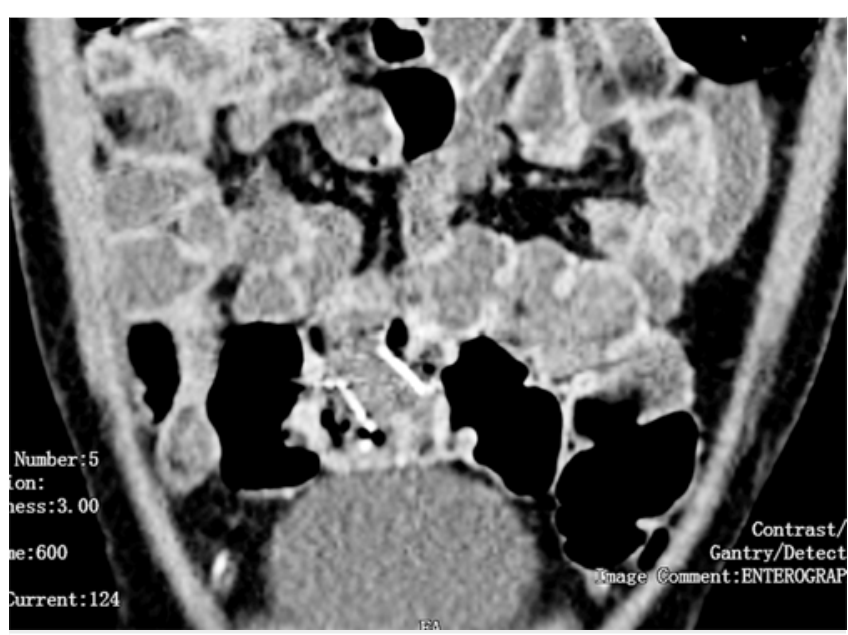

- Fig. 3 FCSEMS migration to rectum on CT of the abdomen.

expelled. Another patient had stent migration to the WON cavity ( $>$ Fig.4 a,b) after 2 weeks and this was withdrawn and reapplied. One patient developed recurrent vomiting and failure to thrive after 6 weeks. Non-contrast computed tomography (NCCT) of the abdomen ( $\mathbf{F i g . 5}$ ) revealed a collapsed cavity, one flange in the cavity and the other flange was obstructing the lumen of the duodenum leading to gastric outlet obstruction (GOO). This stent was removed and the patient became asymptomatic. There were no adverse events related to hydrogen peroxide, i.e. bloating, nausea or bad taste in the mouth. 

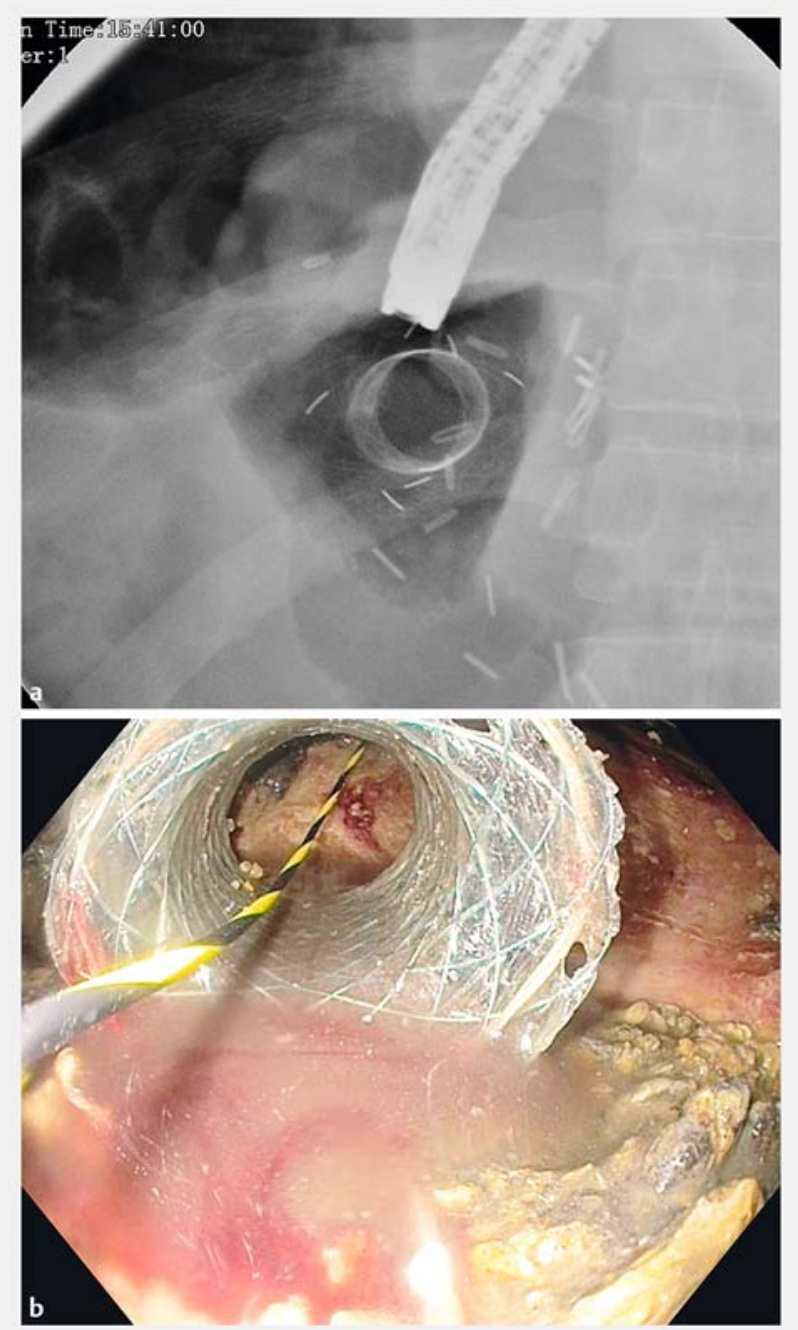

- Fig. 4 a Fluoroscopic image of migrated FCSEMS in WON cavity. b Endoscopic image of migrated FCSEMS in WON cavity.

\section{Follow-up}

Follow-up endoscopy for stent removal was performed 12 weeks after initial drainage after documentation of resolution of WON on NCCT. During removal of the FCSEMS, a pancreatogram was obtained under fluoroscopy.

In $4.7 \%(n=3)$ of patients, plastic stenting of the pancreatic duct was carried out for disruption of the pancreatic duct in the head and body region. In the remaining $95.3 \%(n=61)$ of patients, the pancreatic duct appeared disconnected on the pancreatogram, and no other action was taken.

The mean stenting period was 72 days (range $62-106$ days).

Patients were followed up for 6 months after removal of the FCSEMS both clinically as well as with trans-abdominal ultrasound. All of the patients were asymptomatic and there were no recurrences of WON.

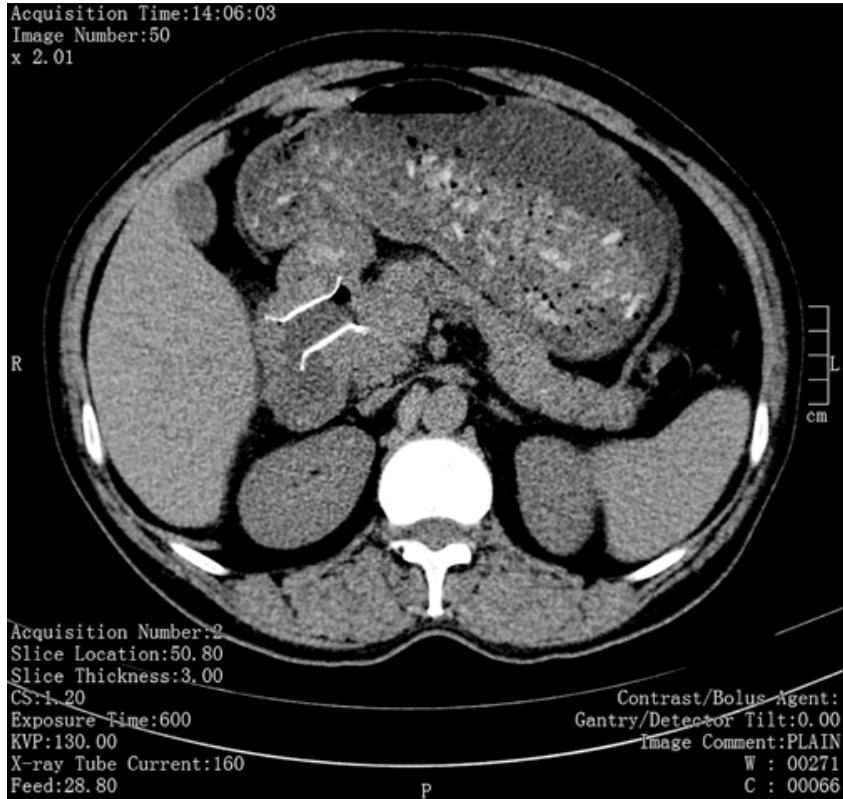

- Fig. 5 FCSEMS causing gastric outlet obstruction on CT of the abdomen.

\section{Discussion}

The present study showed that, in symptomatic WON, endoscopic lavage of a cavity using a large volume of saline solution with hydrogen peroxide and without any debridement is a safe and effective method with a high technical and clinical success rate.

Endoscopic approaches to manage WON have progressed from making small transmural tracts for irrigation to making large tracts that allow the endoscope to move directly into cavities and perform endoscopic necrosectomy, reducing the need for adjuvant PCD [14].

The technical success rate of FCSEMS in WON reported in the literature ranges from $91 \%$ to $100 \%$ and technical failure encountered was related to the technique or device used [9-11, 15]. In our study, FCSEMS were successfully deployed in $100 \%$ of the patients. Successful resolution of WON using FCSEMS has been reported in $75 \%$ to $90 \%$ of patients $[10,11,16]$. In our study, clinical success was achieved in $90.8 \%$, and we could achieve incremental success with each step using the step-up approach.

The clinical success after the index drainage procedure was $28 \%$. The incremental success added with each subsequent step was $72 \%$ for endoscopic necrosectomy by saline irrigation with hydrogen peroxide, and $8 \%$ for PCD insertion. One patient required salvage surgery.

Reported adverse events in pancreatic fluid collection (PFC) drainage using metal stents range from $5 \%$ to $10 \%[16,17]$. The rates of adverse events with dedicated FCSEMS were $5.4 \%$ as reported by Rinninella et al. [16] and $9.8 \%$ by Siddiqui et al. [17]. In our study, adverse events were documented in $12.5 \%$ of patients. Three patients, who developed life threatening bleeding, required special attention. They had documented splenic 
artery pseudoaneurysms on CT angiography. Initial CT did not reveal any pseudoaneurysm. In all three patients, there were significant impingements of the splenic artery by flanges of the FCSEMS and all the stents were long $(30 \mathrm{~mm})$. We assume that the pseudoaneurysms had occurred either because of the disease process or because of the long stent or its shape, so we recommend that short length and smooth flanged FCSEMS are used as they are associated with fewer complications.

The migration rate of metal stents ranged from $0 \%$ to $11 \%$ in various studies. Chandran et al. [10] and Walter et al. [11] reported migration in 4 of 54 and 3 of 61 PFCs, respectively, during direct endoscopic necrosectomy (DEN). Migration of the stent can be either external (into the gastrointestinal lumen) or internal (into the WON cavity). External migration, particularly when associated with resolution of WON, does not require further intervention. For internal migration of a stent, it can be salvaged if detected during the procedure. Clinically significant migration of stents, whether internal or external, can occur occasionally and requires repositioning them to their originally intended site. In this study, stent migration occurred in 3 patients (internal in 1, external in 2), all of which could be salvaged. One stent migration needed special attention. As reported earlier, this rare complication of partial stent migration led to GOO. In this case, the stent was long $(30 \mathrm{~mm})$, and we note that the short stent is associated with fewer complications.

A large number of patients with WON having significant solid debris may require re-intervention because of its non-resolution with or without occlusion of the FCSEMS. Endoscopic reintervention is generally required to remove this clinically significant adherent or non-adherent debris. Non-adherent debris may clog the stent lumen after the fluid within the WON drains out, and may require declogging which can be carried out using various endoscopic devices, especially the snare. Debris adhering to the wall of the WON requires a more intense cleaning approach, known as direct endoscopic necrosectomy (DEN).

There are a lot of published data on the use of DEN and this approach has a high risk of complications $[18,19]$. In a study of 93 patients who underwent direct transluminal endoscopic removal of pancreatic necrotic material (mean of six interventions), there was an $80 \%$ rate of clinical success; however, there was also a $26 \%$ complication rate and a $7.5 \%$ mortality rate at 30 days [18]. In another study of 104 patients, clinical success was achieved in $91 \%$ of patients with a mean time to resolution of 4.1 months after initial DEN. Complications occurred in $14 \%$ of patients and included 5 retrogastric perforations or pneumoperitoneum [19]. In comparison to most published data on FCSEMS for drainage of WON, our method is minimally invasive, as we only performed large volume saline irrigation, without any intracavitary debridement. Smith et al. [2] used $700-$ $1200 \mathrm{~mL}$ of saline for forceful irrigation and lavage using a waterjet. We used almost the same amount of saline, i. e. $1 \mathrm{~L}$ in addition to hydrogen peroxide. Possibly irrigation with hydrogen peroxide and a large volume of saline solution causes liquefaction of necrosis and may also control local sepsis. We believe that repetitive lavage and the presence of a sufficiently large conduit to allow the semiliquid debris to flow out were the key factors for success. This aspect of drainage makes our proce- dure safe as previous studies had significant complications, especially bleeding and local infection secondary to necrosectomy. Our study shows complete resolution of symptomatic WOPN in all of the patients with minimal adverse events.

The removal of FCSEMS is straightforward and uneventful in the majority of patients. The mean stenting period was 72 days (range 62-106 days) and all stents could be safely removed; however, some authors have suggested safe early removal of FCSEMS by 3 weeks to prevent embedding in tissue [20].

Our study had several strengths. First, we followed a systematic step-up approach in managing WON with FCSEMS and demonstrated incremental benefit with each step. Second, by using the less invasive irrigation technique, the aggressive step of DEN and its associated complications can be avoided, and it is also time consuming.

Our study had some limitations. It was a single center study and comparison with established methods was not done. Only a randomized, multicentric comparative study can answer the question whether this method is more efficacious and safer.

We conclude that the step-up approach for the management of symptomatic WON using endoscopic drainage with FCSEMS followed by repeated endoscopic irrigation of the cavity using a saline solution containing hydrogen peroxide, is a minimally invasive, safe, and effective method with a high technical and clinical success rate. We also conclude that long stents are more frequently associated with complications. Although our results are promising, future large and randomized studies are needed to validate our technique.

\section{Competing interests}

None

References

[1] Banks PA, Bollen TL, Dervenis C et al. Acute Pancreatitis Classification Working Group. Classification of acute pancreatitis 2012: revision of the Atlanta classification and definitions by international consensus. Gut 2013; 62: $102-111$

[2] Smith IB, Gutierrez JP, Ramesh J et al. Endoscopic extra-cavitary drainage of pancreatic necrosis with fully covered self-expanding metal stents (fCSEMS) and staged lavage with a high-flow water jet system. Endosc Int Open 2015; 03: E154-E160

[3] Johnson MD, Walsh RM, Henderson JM et al. Surgical versus nonsurgical management of pancreatic pseudocysts. J Clin Gastroenterol 2009; 43: $586-590$

[4] Varadarajulu S, Bang JY, Sutton BS et al. Equal efficacy of endoscopic and surgical cysto-gastrostomy for pancreatic pseudocyst drainage in a randomized trial. Gastroenterology 2013; 145: 583 - 590

[5] Park DH, Lee SS, Moon SH et al. Endoscopic ultrasound-guided versus conventional transmural drainage for pancreatic pseudocysts: a prospective randomized trial. Endoscopy 2009; 41: $842-848$

[6] Baron TH, Harewood GC, Morgan DE et al. Outcome differences after endoscopic drainage of pancreatic necrosis, acute pancreatic pseudocysts, and chronic pancreatic pseudocysts. Gastrointest Endosc 2002; 56: 7-17 
[7] Gornals JB, Consiglieri CF, Busquets J et al. Endoscopic necrosectomy of walled-off pancreatic necrosis using a lumen-apposing metal stent and irrigation technique. Surg Endosc 2016; 30: 2592-2602

[8] Sharaiha RZ, Tyberg A, Khashab MA et al. Endoscopic therapy with lumen-apposing metal stents is safe and effective for patients with pancreatic walled-off necrosis. Clin Gastroenterol Hepatol 2016; 14 : $1797-1803$

[9] Shah R], Shah JN, Waxman L et al. Safety and efficacy of endoscopic ultrasound-guided drainage of pancreatic fluid collections with lumen-apposing covered self-expanding metal stents. Clin Gastroenterol Hepatol 2015; 13: $747-752$

[10] Chandran S, Efthymiou M, Kaffes A et al. Management of pancreatic collections with a novel endoscopically placed fully covered self-expandable metal stent: a national experience (with videos). Gastrointest Endosc 2015; 81: 127 - 135

[11] Walter D, Will U, Sanchez-Yague A et al. A novel lumen-apposing metal stent for endoscopic ultrasound-guided drainage of pancreatic fluid collections: a prospective cohort study. Endoscopy 2015; 47: $63-67$

[12] Thoeni RF. The revised Atlanta classification of acute pancreatitis: its importance for the radiologist and its effect on treatment. Radiology 2012; 262: $751-764$

[13] Gutierrez JP, Wilcox CM, Mönkemüller K. New technique to carry out endoscopic necrosectomy lavage using a pump. Dig Endosc 2014; 26: $117-118$
[14] Baron TH, Kozarek RA. Endotherapy for organized pancreatic necrosis: perspectives after 20 years. Clin Gastroenterol Hepatol 2012; 10: $1202-1207$

[15] Bapaye A, Itoi T, Kongkam P et al. New fully covered large-bore wideflare removable metal stent for drainage of pancreatic fluid collections: results of a multicenter study. Dig Endosc 2015; 27: 499- 504

[16] Rinninella E, Kunda R, Dollhopf $M$ et al. EUS-guided drainage of pancreatic fluid collections using a novel lumen-apposing metal stent on an electrocautery-enhanced delivery system: a large retrospective study (with video). Gastrointest Endosc 2015; 82: 1039-1046

[17] Siddiqui AA, Adler DG, Nieto J et al. EUS-guided drainage of peripancreatic fluid collections and necrosis by using a novel lumen apposing stent: a large retrospective, multicenter U.S. experience. Gastrointest Endosc 2016; 83: 699-707

[18] Seifert H, Biermer M, Schmitt W et al. Transluminal endoscopic necrosectomy after acute pancreatitis: a multicentre study with longterm follow-up (the GEPARD study). Gut 2009; 58: 1260 - 1266

[19] Gardner TB, Coelho-Prabhu N, Gordon SR et al. Direct endoscopic necrosectomy for the treatment of walled-off pancreatic necrosis: results from a multicenter U.S. series. Gastrointest Endosc 2011; 73 : $718-726$

[20] Dhir V, Teoh AY, Bapat M et al. EUS-guided pseudocyst drainage: prospective evaluation of early removal of fully covered self-expandable metal stents with pancreatic ductal stenting in selected patients. Gastrointest Endosc 2015; 82: 650-657 